\title{
Association Between c-Met and Lymphangiogenic Factors in Patients With Colorectal Cancer
}

\author{
Han Jo Kim ${ }^{1, \star}$, Moo-Jun Baek ${ }^{2, *}$, Dong Hyun Kang${ }^{2}$, Sang-Cheol Lee ${ }^{1}$, Sang Byung Bae ${ }^{1}$, Kyu Taek Lee ${ }^{1}$, \\ Namsu Lee ${ }^{3}$, Hyungjoo Kim ${ }^{4}$, Dongjun Jeong 4 , Tae Sung Ahn², Moon Soo Lee ${ }^{2}$, Dae Sik Hong \\ Jong-Ho Won ${ }^{3}$ \\ Departments of ${ }^{1}$ Internal Medicine and ${ }^{2}$ Surgery, Soonchunhyang University Cheonan Hospital, Soonchunhyang University College of \\ Medicine, Cheonan; ${ }^{3}$ Department of Internal Medicine, Soonchunhyang University Seoul Hospital, Soonchunhyang University College of \\ Medicine, Seoul; ${ }^{4}$ Department of Pathology, Soonchunhyang Medical Science Research Institute, Cheonan; ${ }^{5}$ Department of Internal Medicine, \\ Soonchunhyang University Bucheon Hospital, Soonchunhyang University College of Medicine, Bucheon, Korea
}

Purpose: Animal models show a strong relationship between lymphangiogenesis and lymph node metastasis. However, the clinical significance of lymphangiogenesis in patients with colorectal cancer (CRC) remains uncertain. This study aimed to evaluate the association between c-Met and lymphangiogenic factors and to elucidate the prognostic significance of c-Met in patients with CRC.

Methods: A total of 379 tissue samples were obtained from surgically resected specimens from patients with CRC at Soonchunhyang University Cheonan Hospital between January 2002 and December 2010. The expressions of c-Met, vascular endothelial growth factor (VEGF)-C, VEGF-D, VEGF receptor (VEGFR)-3, and podoplanin were examined using immunohistochemistry. The expression of c-Met and clinical factors were analyzed.

Results: Of the 379 tissues, 301 (79.4\%) had c-Met expression. High expression of c-Met in tumor cells was significantly associated with high expression of VEGF-C $(\mathrm{P}<0.001)$ and VEGFR-3 $(\mathrm{P}=0.001)$. However, no statistically significant association with podoplanin $(\mathrm{P}=0.587)$ or VEGF-D $(\mathrm{P}=0.096)$ was found. Of the 103 evaluable patients, expression of $\mathrm{c}$ Met in tumor cells was significantly associated with advanced clinical stage $(\mathrm{P}=0.020)$, positive lymph node status $(\mathrm{P}=$ $0.038)$, and high expression of VEGF-C $(\mathrm{P}=0.020)$. However, no statistically significant association with podoplanin $(\mathrm{P}=$ 0.518), VEGFR-3 ( $\mathrm{P}=0.085)$, VEGF-D ( $\mathrm{P}=0.203)$, or overall survival $(\mathrm{P}=0.360)$ was found.

Conclusion: Our results provide indirect evidence for an association and possible regulatory link of c-Met with the lymphangiogenic markers, but c-Met expression in patients with CRC is not a prognostic indicator for overall survival.

Keywords: Lymphangiogenesis; c-Met; Colorectal neoplasms

Received: September 6, 2017 - Accepted: October 10, 2017

Correspondence to: Jong-Ho Won, M.D.

Department of Internal Medicine, Soonchunhyang University Seoul Hospital, Soonchunhyang University College of Medicine, 59 Daesagwan-ro,

Yongsan-gu, Seoul 04401, Korea

Tel: +82-2-709-9203, Fax: +82-2-709-9200

E-mail: jhwon@schmc.ac.kr

ORCID code: https://orcid.org/0000-0001-6176-1442

*Han Jo Kim and Moo-Jun Baek contributed equally to this study as co-first authors.

(C) 2018 The Korean Society of Coloproctology

This is an open-access article distributed under the terms of the Creative Commons Attribution NonCommercial License (http://creativecommons.org/licenses/by-nc/4.0) which permits unrestricted noncommercial use, distribution, and reproduction in any medium, provided the original work is properly cited.

\section{INTRODUCTION}

Colorectal cancer $(\mathrm{CRC})$ is a major health problem and one of the leading causes of cancer-related death worldwide [1]. Patients with lymphatic invasion have a less favorable outcome, and lymph node metastasis is a very important prognostic factor in CRC [2]. Although the pattern of spread of CRC may vary, the initial step involves lymphatic invasion and metastasis to regional lymph nodes [3].

Meanwhile, several growth factors have been found to contribute to lymphangiogenesis in solid tumors; these include vascular endothelial growth factor (VEGF) C, VEGF-D, VEGF receptor-3 (VEGFR-3), podoplanin, and c-Met [4]. VEGF-C and -D have 
been identified as specific lymphangiogenic factors that act via activation of VEGFR-3, which is expressed in lymphatic endothelial cells [5]. Hepatocyte growth factor (HGF) is a heparin-binding glycoprotein produced by various cells of mesenchymal origin. In vivo studies have shown that HGF plays an important role in tissue repair and promotes tumor invasiveness [6]. c-Met, as the receptor of HGF, was found to be overexpressed in various types of tumors, mediating its multiple roles, such as promoting tumor cell growth, including tumor cell invasion, and stimulating angiogenesis [7].

Recently, in experimental cancer metastasis models, a growing amount of evidence has been found that tumor lymphangiogenesis may further facilitate tumor metastases. However, the clinical significance of lymphangiogenesis in patients with CRC remains uncertain. The aim of this study was to evaluate the association between c-Met and lymphangiogenic factors and to elucidate the prognostic significance of c-Met in patients with CRC.

\section{METHODS}

Three hundred seventy-nine patients with CRC, who were diagnosed and surgically treated at Soonchunhyang University Hospital between January 2002 and December 2010, were enrolled in this study. The clinical variables, including sex, age, and tumor stage were all obtained preoperatively. Surgical specimens were evaluated for histopathologic staging. The patients were classified according to the 6th edition of the American Joint Committee on Cancer Staging System [8]. The expressions of c-Met, VEGF-C, VEGF-D, VEGFR-3, and podoplanin were examined by using immunohistochemistry (IHC). The expression of c-Met and the clinical factors was analyzed. Our study was approved by the Clinical Ethics Review Committee at Soonchunhyang University Hospital, Cheonan, Republic of Korea (approval number: 201508-023). Written informed clinical consent was obtained from all the patients.

For construction of the tissue microarrays (TMAs), areas representative of cancer were marked on slides stained with hematoxylin and eosin (H\&E), and TMAs were constructed. TMAs were created from formalin-fixed (10\% neutral buffered formalin), paraffin-embedded tissues by using a 2 -mm diameter punch (UNITMA, Unitech Science, Seoul, Korea). TMA blocks were assembled by getting duplicate cores from one patient block and reembedding the 2 cores in an arrayed recipient block (UNITMA, Unitech Science). A TMA block contains 60 cores from 30 samples.

In the preparation for IHC staining, the TMAs were sectioned at 4-micron intervals, deparaffinized three times in xylene for 30 minutes, rehydrated with graded alcohol (100\% ethyl alcohol for 5 minutes, 95\% ethyl alcohol for 3 minutes, and 75\% ethyl alcohol for 3 minutes), and then heated in antigen-retrieval solution (sodium citrate, $\mathrm{pH}$ 6.0) in a microwave for 20 minutes. Sections were incubated in $\mathrm{H}_{2} \mathrm{O}_{2}$ for 10 minutes at room temperature. Next, the sections were incubated with $150 \mathrm{~mL}$ of the primary an- tibodies, c-Met (1:50, AbFrontier, Seoul, Korea, \#LF-PA20708), VEGF-C (1:200, R\&D systems, Minneapolis, MN, USA, \#AF752), VEGF-D (1:100, Abcam plc, Cambridge, UK, \#ab103685), VEGFR-3 (1:50, Abcam plc, \#ab72240), and podoplanin (1:200, ReliaTech GmbH, Wolfenbüttel, Germany, \#101-M41), at $4^{\circ} \mathrm{C}$ overnight. The sections were then washed in phosphate buffered saline (PBS) buffer three times for 3 minutes, treated with $150 \mathrm{~mL}$ of secondary antibody for 1 hour at room temperature, and stained with DAB solution (Dako, Carpinteria, CA, USA). The sections were then washed in PBS buffer for 10 minutes. Finally, the sections were counterstained with hematoxylin for 3 minutes at room temperature, washed in distilled water 3 times for $3 \mathrm{~min}$ utes, and mounted on coverslips.

For the IHC analysis, the c-Met, VEGF-C, VEGF-D, VEGFR-3, and podoplanin stained tissue cores were examined by 2 independent pathologists, and a consensus score was determined for each specimen. A positive reaction for both antibodies was scored into 4 grades according to the intensity of the staining: $0,1+, 2+$, and $3+$. The percentages of positive cells were also scored into four categories: $0(0 \%), 1(1 \%-33 \%), 2(34 \%-66 \%)$, and $3(67 \%-$ $100 \%)$. The final score, calculated as the product of the intensity and the percentage score, was classified as follows: 0 for negative, 1-3 for weak, 4-6 for moderate, and 7-9 for strong. Then, we recategorized negative \& weak positive into low c-Met expression and moderate \& strong positive into high c-Met expression.

For the statistical analysis, the correlations between c-Met and lymphangiogenic factors was evaluated by using the chi-square or Fisher exact test. The Kaplan-Meier method was used to generate overall survival curves, and differences between cohorts were tested using log-rank statistics. All P-values quoted were 2-sided, and $\mathrm{P}<0.05$ was considered statistically significant. All the analyses were performed using SPSS ver. 17.0 (SPSS Inc., Chicago, IL, USA).

\section{RESULTS}

The median age of the 379 patients with CRC was 55 years (range, 24-88 years). By sex, 219 (57.8\%) were male, and 160 (42.2\%) were female patients. On the c-Met expression profiles, c-Met immunohistostaining positivity was observed in the cytoplasm and the cell membrane as brown staining (Fig. 1). Cases with high cMet expression outnumbered cases showing low c-Met expression: 191 cases (50.4\%) of high expression and 188 cases (49.6\%) of low expression (Table 1). No significant correlation between cMet expression and age, sex, tumor stage (depth of invasion, nodal metastasis, and distant metastasis) or lymphovascular invasion was found (Table 2). High expression of c-Met in tumor cells was significantly associated with high expression of VEGF-C $(\mathrm{P}<$ $0.001)$ and VEGFR-3 $(P=0.001)$, but no statistically significant association with podoplanin $(\mathrm{P}=0.587)$ or VEGF-D $(\mathrm{P}=0.096)$ was found (Table 3).

For the 103 patients for whom a survival analysis could be done, 


\begin{aligned} & Annals of $\begin{array}{l}\text { Association Between c-Met and Lymphangiogenic Factors in Patients With Colorectal Cancer } \\ \text { Han Jo Kim, et al. }\end{array} \\ &$\hline\end{aligned}
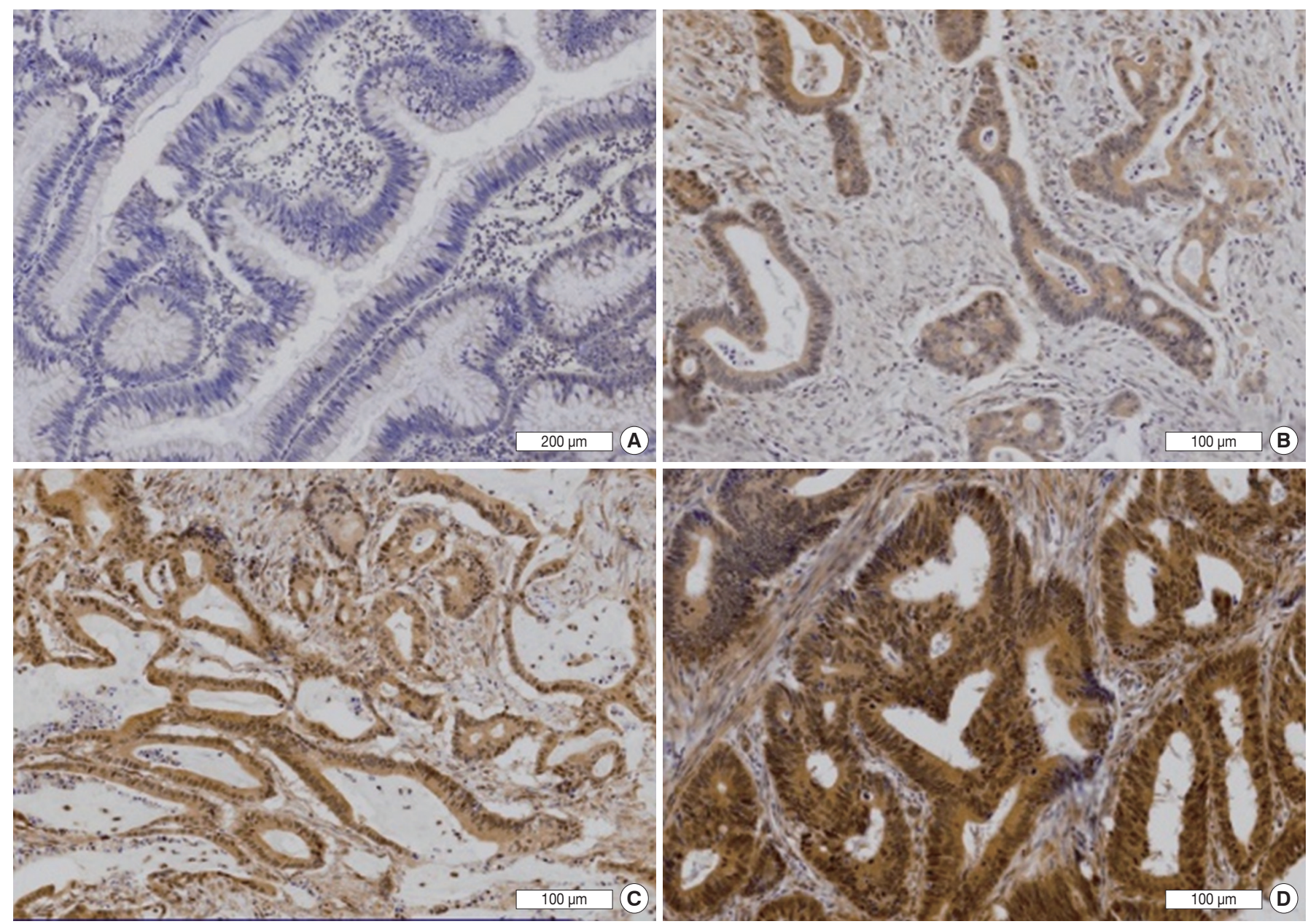

Fig. 1. Overexpression of c-Met: negative expression (A), 1 positive status (B), 2 positive status (C), and 3 positive status (D) based on the strength of immunohistochemistry staining $(\mathrm{H} \& \mathrm{E}, \times 200)$. c-Met immunohistostaining positivity was observed in the cytoplasm and the cell membrane as brown staining.

Table 1. c-Met expression profiles

\begin{tabular}{lc}
\hline c-Met expression & No. (\%) \\
\hline 0 & $78(20.6)$ \\
1 & $110(29.0)$ \\
2 & $161(42.5)$ \\
3 & $30(7.9)$ \\
Total & $379(100)$ \\
\hline
\end{tabular}

expression of c-Met in tumor cells was significantly associated with advanced clinical stage $(\mathrm{P}=0.020)$, positive lymph node status $(\mathrm{P}=0.038)$, and high expression of VEGF-C $(\mathrm{P}=0.020)$. However, no statistically significant association with podoplanin $(\mathrm{P}=0.518)$, VEGFR-3 $(\mathrm{P}=0.085)$, VEGF-D $(\mathrm{P}=0.203)$, or overall survival $(\mathrm{P}=0.360$ ) was found (Fig. 2 , Table 4$)$. We also performed survival analyses after having categorized c-Met was as high and low expression and found no correlation with survival (P $=0.805)$ (Fig. 3).

\section{DISCUSSION}

In a variety of human cancers, tumor metastasis to regional lymph nodes represents the first step of dissemination and serves as a prognostic implication. The extent of lymph node metastasis is a critical determinant for cancer staging and prognosis, which often guides therapeutic decisions. However, in patients with CRC, the molecular mechanism of lymphatic metastasis is not completely understood, and the role of the c-Met signaling pathway has not been fully elucidated. With this theoretical background, we investigated the expressions of c-Met and several lymphangiogenic factors (VEGF-C, VEGF-D, VEGFR-3, podoplanin) in surgical specimens from 379 patients with CRC to evaluate their clinical significance and the associations between c- 
Table 2. Association between clinicopathological features and c-Met expression

\begin{tabular}{|c|c|c|c|c|}
\hline \multirow[b]{2}{*}{ Clinicopathological factors } & \multicolumn{2}{|c|}{ c-Met } & \multirow[b]{2}{*}{ Total } & \multirow[b]{2}{*}{ P-value } \\
\hline & $\begin{array}{l}\text { Positive } \\
(n=301)\end{array}$ & $\begin{array}{l}\text { Negative } \\
(n=78)\end{array}$ & & \\
\hline Median age (yr) & 55 & 56 & & \\
\hline Sex & & & & 0.191 \\
\hline Male & $190(63.0)$ & $29(36.8)$ & $219(57.8)$ & \\
\hline Female & $111(37.0)$ & 49 (63.2) & $160(42.2)$ & \\
\hline pT stage & & & & 0.120 \\
\hline \multicolumn{5}{|l|}{1} \\
\hline 2 & $56(18.5)$ & $8(10.5)$ & $64(16.9)$ & \\
\hline 3 & $212(70.3)$ & $59(75.4)$ & $271(71.5)$ & \\
\hline 4 & $33(11.2)$ & $11(14.1)$ & $44(11.6)$ & \\
\hline pN stage & & & & 0.143 \\
\hline 0 & $100(33.3)$ & $33(42.2)$ & $133(35.1)$ & \\
\hline 1,2 & $201(66.7)$ & $45(57.8)$ & $246(64.9)$ & \\
\hline Vascular invasion & & & & 0.252 \\
\hline Absent & $256(85.2)$ & $66(84.2)$ & $322(85.0)$ & \\
\hline Present & $45(14.8)$ & $12(15.8)$ & $57(15.0)$ & \\
\hline Lymphatic invasion & & & & 0.204 \\
\hline Absent & $233(74.1)$ & $56(71.9)$ & $289(76.3)$ & \\
\hline Present & $68(25.9)$ & $22(28.1)$ & $90(23.7)$ & \\
\hline Metastasis & & & & 0.452 \\
\hline 0 & $290(96.3)$ & 75 (96.5) & $365(96.3)$ & \\
\hline 1 & $11(3.7)$ & $3(3.5)$ & $14(3.7)$ & \\
\hline TNM stage & & & & 0.143 \\
\hline I, II & $100(33.3)$ & $33(42.1)$ & $133(35.1)$ & \\
\hline III, IV & $201(66.7)$ & $45(57.9)$ & $246(64.9)$ & \\
\hline
\end{tabular}

Values are presented as number (\%).

Met and lymphangiogenic factors.

Forced expression of VEGF-C in xenografts and in transgenic tumors results in tumor lymphangiogenesis and increased tumor dissemination to regional lymph nodes [9-11]. In another study, the inhibition of the VEFGR-3 pathway, either VEGF-C/D trap or VEGFR-3 blocking antibodies suppressed approximately $60 \%-$ $70 \%$ of lymph node metastasis in a variety of experimental tumor models [12-15]. In this study, we observed that a high expression of c-Met in tumor cells was significantly associated with high expressions of VEGF-C $(\mathrm{P}<0.001)$ and VEGFR-3 $(\mathrm{P}=0.001)$. From this point of view, we believe that the results from animal studies have been proven by our study using clinical specimen, although we found no correlations with podoplanin and VEGF-D.

c-Met is overexpressed in a variety of carcinomas, including breast, lung, gastric and colon carcinomas [16-18]. In previous reports, the proportion of c-Met expression was 78\%. Similar results
Table 3. Association between c-Met and lymphangiogenic markers

\begin{tabular}{|c|c|c|c|c|}
\hline \multirow{2}{*}{ Marker } & \multicolumn{2}{|c|}{ c-Met } & \multirow{2}{*}{ Total } & \multirow{2}{*}{ P-value } \\
\hline & Low expression (L) & High expression $(\mathrm{H})$ & & \\
\hline VEGF-C & & & & $<0.001$ \\
\hline L & 159 & 130 & 289 & \\
\hline $\mathrm{H}$ & 29 & 61 & 90 & \\
\hline VEGF-D & & & & 0.096 \\
\hline L & 146 & 134 & 280 & \\
\hline $\mathrm{H}$ & 42 & 57 & 99 & \\
\hline VEGFR-3 & & & & 0.001 \\
\hline L & 181 & 166 & 347 & \\
\hline $\mathrm{H}$ & 7 & 25 & 32 & \\
\hline Podoplanin & & & & 0.587 \\
\hline L & 118 & 125 & 243 & \\
\hline $\mathrm{H}$ & 70 & 66 & 136 & \\
\hline
\end{tabular}

VEGF, vascular endothelial growth factor; VEGFR, VEGF receptor.

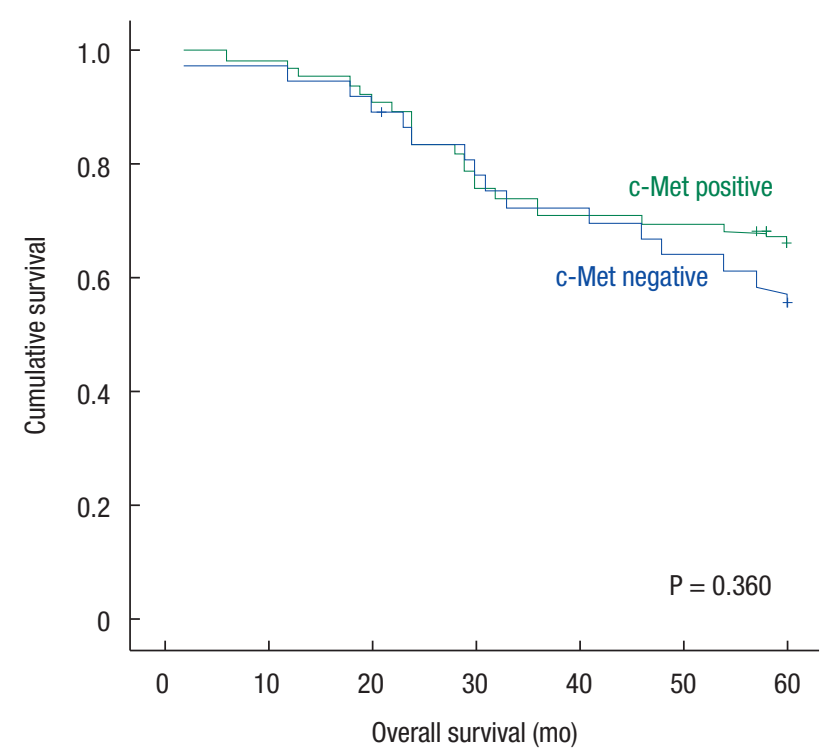

Fig. 2. Kaplan-Meier survival curves for c-Met expression (positive vs. negative) in patients with colorectal cancer $(\mathrm{n}=103)$. No statistically significant association with overall survival was observed $(\mathrm{P}=0.360)$.

were found in the present study; c-Met expression in patients with CRC was 79.4\% (Table 1). Also, high levels of c-Met and/or HGF expression have been associated with poor survival outcome in a variety of carcinomas, including CRC [17, 19]. However, contrary to our expectation, the present data showed that c-Met expression was not associated with $\mathrm{CRC}$ prognosis $(\mathrm{P}=0.360)$ (Fig. 2). This finding may have been due to the fact that our investigation included more patients than the previous studies did $[17,19]$. If the correlation of prognosis with c-Met expression is to be accurately determined, an additional study with a large cohort of patients in 
Table 4. Association between clinicopathological features and c-Met expression in patients for whom a survival analysis was performed $(\mathrm{n}=103)$

\begin{tabular}{|c|c|c|c|}
\hline \multirow[b]{2}{*}{ Clinicopathological factors } & \multicolumn{2}{|c|}{ c-Met } & \multirow[b]{2}{*}{ P-value } \\
\hline & $\begin{array}{l}\text { Positive } \\
(n=66)\end{array}$ & $\begin{array}{l}\text { Negative } \\
(\mathrm{n}=37)\end{array}$ & \\
\hline pT stage & & & 0.146 \\
\hline 1,2 & 17 & 5 & \\
\hline 3,4 & 49 & 32 & \\
\hline pN stage & & & 0.038 \\
\hline 0 & 39 & 14 & \\
\hline 1,2 & 27 & 23 & \\
\hline TNM stage & & & 0.020 \\
\hline I, II & 39 & 13 & \\
\hline III, IV & 27 & 24 & \\
\hline Podoplanin & & & 0.518 \\
\hline Negative & 40 & 20 & \\
\hline Positive & 26 & 17 & \\
\hline VEGFR-3 & & & 0.085 \\
\hline Negative & 60 & 37 & \\
\hline Positive & 6 & 0 & \\
\hline VEGFD & & & 0.203 \\
\hline Negative & 48 & 31 & \\
\hline Positive & 18 & 6 & \\
\hline
\end{tabular}

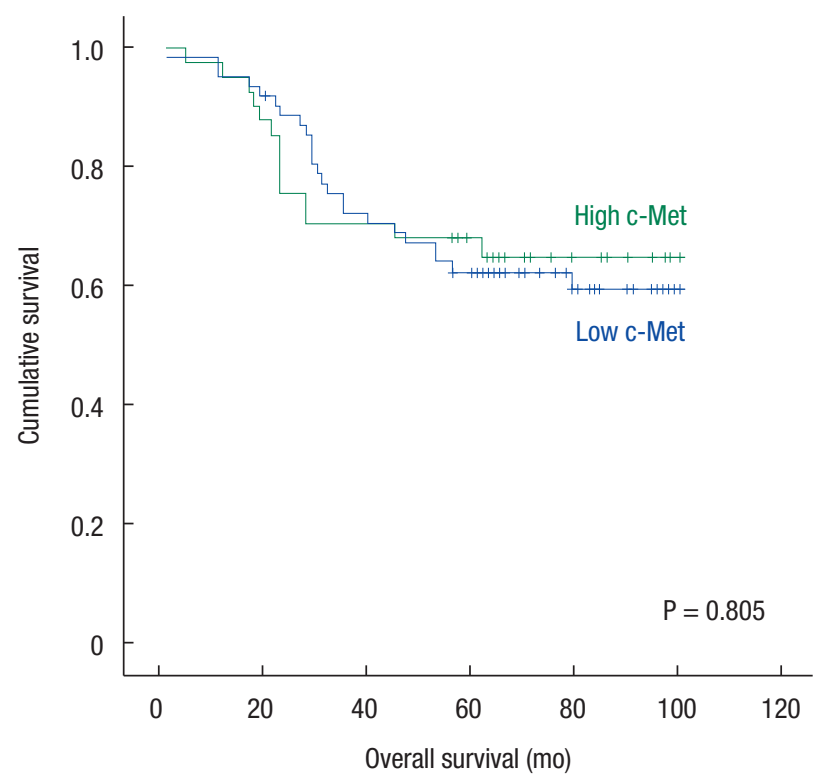

Fig. 3. Kaplan-Meier survival curves for c-Met expression (high vs. low) in patients with colorectal cancer $(\mathrm{n}=103)$. No statistically significant association with overall survival was observed $(\mathrm{P}=0.805)$. whom c-Met expression was observed is necessary.

The limitations of the present study include its retrospective design. The study could have been affected by potential selection bias. For example, $\mathrm{c}$-Met did not correlate with tumor stage $(\mathrm{n}=$ $379)$; however, it did correlate with tumor stage $(n=103)$ in patients for whom a survival analysis was performed. Another limitation is the lack of cancer-specific survival. In conclusion, the major finding of this study was that indirect evidence exists for an association and possible regulatory link of c-Met with the lymphangiogenic markers. Nevertheless, c-Met expression in patients with CRC is not a prognostic indicator for overall survival.

\section{CONFLICT OF INTEREST}

No potential conflict of interest relevant to this article was reported.

\section{ACKNOWLEDGMENTS}

This research was supported by a grant of the Korea Health Technology R\&D Project through the Korea Health Industry Development Institute (KHID), funded by the Ministry of Health \& Welfare, Republic of Korea (grant number: HI17C0031). This work was also supported by the Soonchunhyang University Research Fund.

\section{REFERENCES}

1. Siegel R, Naishadham D, Jemal A. Cancer statistics, 2012. CA Cancer J Clin 2012;62:10-29.

2. Vather R, Sammour T, Kahokehr A, Connolly AB, Hill AG. Lymph node evaluation and long-term survival in Stage II and Stage III colon cancer: a national study. Ann Surg Oncol 2009;16: 585-93.

3. Sundlisaeter E, Dicko A, Sakariassen PØ, Sondenaa K, Enger PØ, Bjerkvig R. Lymphangiogenesis in colorectal cancer--prognostic and therapeutic aspects. Int J Cancer 2007;121:1401-9.

4. Zwaans BM, Bielenberg DR. Potential therapeutic strategies for lymphatic metastasis. Microvasc Res 2007;74:145-58.

5. Jussila L, Alitalo K. Vascular growth factors and lymphangiogenesis. Physiol Rev 2002;82:673-700.

6. Rosen EM, Knesel J, Goldberg ID, Jin L, Bhargava M, Joseph A, et al. Scatter factor modulates the metastatic phenotype of the EMT6 mouse mammary tumor. Int J Cancer 1994;57:706-14.

7. Jiang WG, Martin TA, Parr C, Davies G, Matsumoto K, Nakamura T. Hepatocyte growth factor, its receptor, and their potential value in cancer therapies. Crit Rev Oncol Hematol 2005;53:35-69.

8. Greene FL, Page DL, Fleming ID, Fritz AG, Balch CM, Haller DG, et al., editors. AJCC cancer staging manual. 6th ed. New York: Springer-Verlag; 2002.

9. Achen MG, McColl BK, Stacker SA. Focus on lymphangiogenesis in tumor metastasis. Cancer Cell 2005;7:121-7. 
10. Alitalo K, Tammela T, Petrova TV. Lymphangiogenesis in development and human disease. Nature 2005;438:946-53.

11. Tobler NE, Detmar M. Tumor and lymph node lymphangiogenesis: impact on cancer metastasis. J Leukoc Biol 2006;80:691-6.

12. Burton JB, Priceman SJ, Sung JL, Brakenhielm E, An DS, Pytowski B, et al. Suppression of prostate cancer nodal and systemic metastasis by blockade of the lymphangiogenic axis. Cancer Res 2008;68:7828-37.

13. He Y, Rajantie I, Pajusola K, Jeltsch M, Holopainen T, Yla-Herttuala S, et al. Vascular endothelial cell growth factor receptor 3-mediated activation of lymphatic endothelium is crucial for tumor cell entry and spread via lymphatic vessels. Cancer Res 2005;65: 4739-46.

14. Lin J, Lalani AS, Harding TC, Gonzalez M, Wu WW, Luan B, et al. Inhibition of lymphogenous metastasis using adeno-associated virus-mediated gene transfer of a soluble VEGFR-3 decoy receptor. Cancer Res 2005;65:6901-9.

15. Roberts N, Kloos B, Cassella M, Podgrabinska S, Persaud K, Wu Y, et al. Inhibition of VEGFR-3 activation with the antagonistic antibody more potently suppresses lymph node and distant metastases than inactivation of VEGFR-2. Cancer Res 2006;66:2650-7.

16. Ma PC, Tretiakova MS, MacKinnon AC, Ramnath N, Johnson C, Dietrich S, et al. Expression and mutational analysis of MET in human solid cancers. Genes Chromosomes Cancer 2008;47:102537.

17. Kammula US, Kuntz EJ, Francone TD, Zeng Z, Shia J, Landmann $\mathrm{RG}$, et al. Molecular co-expression of the c-Met oncogene and hepatocyte growth factor in primary colon cancer predicts tumor stage and clinical outcome. Cancer Lett 2007;248:219-28.

18. Seiden-Long IM, Brown KR, Shih W, Wigle DA, Radulovich N, Jurisica I, et al. Transcriptional targets of hepatocyte growth factor signaling and Ki-ras oncogene activation in colorectal cancer. Oncogene 2006;25:91-102.

19. Toiyama Y, Miki C, Inoue Y, Okugawa Y, Tanaka K, Kusunoki M. Serum hepatocyte growth factor as a prognostic marker for stage II or III colorectal cancer patients. Int J Cancer 2009;125:1657-62. 